Ophthalmologe 2012 · 109:1062-1063

DOI 10.1007/s00347-012-2636-6

Online publiziert: 21. November 2012

c) Springer-Verlag Berlin Heidelberg 2012

G.K. Krieglstein

Univ. Augenkinik, Köln

\title{
Konservanzien in Ophthalmika
}

dex" (Relation von Nutzen zu Risiken) lässt sich im Falle der Konservanzien keiner metrischen Betrachtung unterziehen, zu groß ist die interindividuelle Variabilität der Risiken - Therapiedauer, individuelle Empfindlichkeit des äußeren Auges, Mehrfachtherapie, Umwelteinflüsse, Operationsanamnese etc. Dies verlangt eine Abwägung in der jeweiligen Situation - ist eine Operation geplant, besteht eine Anamnese einer chronischen Konjunktivitis oder einer okulären Allergie, liegt ein Kontaktlinsenbedarf vor, bestehen objektive Befunde einer Meibom-Dysfunktion u. a. Insbesondere bei einem chronischen Therapieanspruch am äußeren Auge über viele Jahre hinweg wie beim Glaukom bedarf die Problematik der Belastung der Oberflächenqualität des äußeren Auges durch Konservanzien einer sorgfältigen, bedarfsgerechten, individuellen Abwägung. Die folgenden Artikel möchten dazu beitragen, die klinische Relevanz der Problematik verständlich zu machen, Entscheidungshilfen zu geben, besondere Vorsichtsparameter bei der langfristigen Anwendung von Konservanzien aufzuzeigen und einen Blick auf die Ökonomie zu werfen, wenn auf Konservanzien zu verzichten ist.

So wird in dem kritischen Beitrag von E.M. Messmer, München, zu den gebräuchlichen Konservierungsstoffen in der Augenheilkunde überzeugend dargestellt, dass die eingesetzten Konservanzien sich sehr wohl in ihren bakteriziden Eigenschaften wie auch in ihren Risikoprofilen unterscheiden durchaus relevant für die klinische
Praxis. Die Autoren K.K. Huber-van der Velden, Düsseldorf, und M. Eichhorn, Erlangen, geben eine Übersicht zu den morphologischen Befunden, die durch Konservanzien ausgelöst werden können - wichtig für die korrekte Einschätzung von Symptom und klinischem Befund. Häufig unterschätzt wird die Interaktion von Oberflächenveränderungen des Auges, ausgelöst durch Konservierungsstoffe, und den Einflüssen auf die Glaukomdiagnostik. Der Beitrag der Autoren F. Rüfer, Kiel, und C. Erb, Berlin, schafft hierzu Klarheit. Änderungen des Oberflächenmilieus des Auges mit entsprechenden Symptomen kann die Therapietreue des Patienten bei einer chronischen Therapie durchaus tangieren. Der Beitrag von I. Lanzl, München, und T. Kaercher, Heidelberg, beleuchten dies in ihrem Beitrag. Konservanzien können die zelluläre Qualität der subkonjunktivalen Bindehaut ändern und damit die postoperative Wundreparation nach Glaukomchirurgie beschleunigen, d. h. die Vernarbung eines Filterkissens fördern (Beitrag H. Thieme, Mainz, und K.K. Huber-van der Velden, Düsseldorf). Eine Problemreduktion durch die Anwendung unkonservierter Einmaldosen muss nicht zwangsläufig die Behandlungskosten explodieren lassen (S. Schneider, Bayreuth).

Die Anwendung von Konservanzien in Arzneilösungen zur Anwendung am äußeren Auge hat viele Facetten. Eine große Anzahl wird hier aufgezeigt und diskutiert. Die klinische Relevanz für die augenärztliche Praxis ist unstrittig, 
die kritische Auseinandersetzung wichtig für die tägliche augenärztliche Praxis.

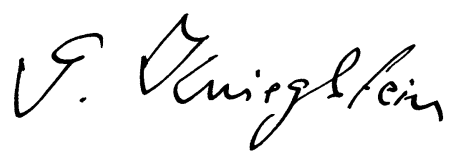

Prof. emer. Dr. G.K. Krieglstein

\section{Korrespondenzadresse

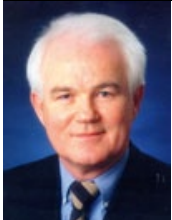 \\ Prof. emer. Dr. G.K. Krieglstein \\ Univ. Augenkinik 50924 Köln \\ guenter.krieglstein@ \\ uk-koeln.de}

Interessenkonflikt. Der korrespondierende Autor weist auf folgende Beziehung hin: Mitglied Advisory Board MSD.

\section{Galenus-von- Pergamon-Preis 2012}

\section{Die Jury hat entschieden}

Für manche ist er der inoffizielle „Nobelpreis" für Pharmakologie: der Galenus-von-Pergamon-Preis. Am 18. Oktober wurde er im Rahmen einer festlichen Gala erneut verliehen - an drei beeindruckende Preisträger.

Primary Care

Der Preis in der Kategorie Primary Care würdigt ein Medikament, das bei einer breiten Patientengruppe eingesetzt wird. In diesem Jahr hat Novartis Pharma diesen Preis für Gilenya ${ }^{\circledR}$ (Fingolimod) erhalten. Fingolimod ist ein orales Medikament zur Therapie von Patienten mit Multipler Sklerose (MS). Es ist zugelassen für bisher nicht behandelte Patienten, die an einer rasch fortschreitenden, schweren schubförmigen MS erkrankt sind. Es ist zudem indiziert zur Eskalationstherapie, wenn trotz Behandlung mit einem Beta-Interferon eine hohe Krankheitsaktivität vorliegt. Mit Fingolimod gelingt es, die Schubrate zu verringern.

Specialist Care

Der Preis in der Kategorie Specialist Care zeichnet ein Medikament aus, das zur Behandlung seltener Erkrankungen verwendet wird. Der diesjährige Gewinner ist Zelboraf ${ }^{\circledast}$ (Vemurafenib) von Roche Pharma. Vemurafenib ist die erste Option für eine personalisierte Therapie bei inoperablem oder metastasiertem Melanom. Das Medikament in Form von Filmtabletten ist zugelassen zur Therapie von Melanompatienten, die ein mutiertes BRAF-Gen haben. In der Zulassungsstudie betrug die geschätzte mediane progressionsfreie Überlebenszeit 5,6 Monate im Vergleich zu Patienten mit der Standardchemothera- pie mit Dacarbazin. In der VemurafenibGruppe war zudem das Sterberisiko um $63 \%$ und das Progressionsrisiko um 74\% verringert.

\section{Grundlagenforschung}

In der Kategorie Grundlagenforschung wurde das Team um Dr. Thomas Worzfeld aus Bad Nauheim für die Entwicklung eines neuen Ansatzes zur Therapie bei metastasierendem Brustkrebs geehrt. Worzfeld und sein Team haben herausgefunden, dass der Rezeptor Plexin-B1 eine besondere Bedeutung für die Metastasierung bei Brustkrebs hat. Anhand von Gewebeproben von Patientinnen mit einem Mammakarzinom stellten sie fest, dass die Frauen eine umso bessere Überlebenschance hatten, je weniger Rezeptoren im Tumorgewebe vorhanden waren. Inzwischen steht ein monoklonaler Antikörper gegen diesen Rezeptor zur Verfügung, der derzeit präklinisch getestet wird.

\section{Springer Medizin CharityAward 2012}

Das Kinder- und Jugendhospiz Balthasar in Olpe ist für sein unermüdliches Engagement mit dem Springer Medizin CharityAward ausgezeichnet worden. Balthasar wurde im Jahr 1998 gegründet und ist damit das erste Kinder-und Jugendhospiz in Deutschland. Der von Schirmherr Daniel Bahr überreichte Springer Medizin CharityAward umfasst einen Barscheck über 50.000 Euro und ein Medienpaket über weitere 100.000 Euro. Damit unterstützt die Fachverlagsgruppe den Gewinner gezielt bei seiner Öffentlichkeitsarbeit.

Quelle: Springer Medizin 